\title{
Can we improve the statistical analysis of vascular prevention trials?: results from the Optimising the analysis of vascular Prevention trials (OA-Prevention) collaboration
}

Lisa J Woodhouse ${ }^{1}$, Alan Montgomery ${ }^{2}$, Philip M Bath ${ }^{1}$; for the OA-Prevention collaborators

1 Stroke, Division of Clinical Neuroscience, University of Nottingham, Nottingham 2 Nottingham Clinical Trials Unit, University of Nottingham, Nottingham Email: L.Woodhouse@nottingham.ac.uk

\section{BACKGROUND}

- Vascular prevention trials typically use binary event outcomes, although this is inefficient statistically and gives no indication of severity; outcomes could therefore be polychotomised, with ordering determined by severity.

- OA-Prevention aims to identify the most efficient type of outcome and analysis technique for vascular prevention data in randomised controlled trials (RCTs).

\section{METHOD}

- Eligible RCTs were identified using search engines such as the Cochrane Library and PubMed.

- Chief investigators of these trials were invited to share individual patient data with the collaboration: data requested included trial characteristics, patient demographics and outcome measures.

- Extensions of this outcome were analysed using various statistical techniques (ordinal logistic regression, Chi-square test and t-test) with and without adjustment (adjusted for age, sex and history of diabetes).

- Results of these analyses were then ordered within each trial set and given a rank; the most significant result (smallest $p$-value) was given the lowest rank.

- The Freidman and Duncan's multiple range tests were then performed to assess significant differences between tests.

\section{RESULTS (Continued)}

- In a direct comparison, analyses performed on this extended outcome (e.g. MWU, ordinal logistic regression) were more efficient than those performed on a binary stroke/no-stroke outcome $(p<0.001)$.

- Table 1 shows the results from the Duncan's multiple range test.

- The most efficient was the MWU test with a mean rank of 4.69 .

\section{Table 1}

\begin{tabular}{|c|c|c|}
\hline Test* & Grouping & Mean \\
\hline MWU 4 & & 4.69 \\
\hline OLR 4 & & 6.26 \\
\hline Adjusted OLR 4 & & 6.31 \\
\hline Bootstrapping & & 6.43 \\
\hline Win Ratio & & 6.69 \\
\hline CA Trend 4 & & 6.71 \\
\hline t-test & & 6.89 \\
\hline Adjusted MR 4 & & 6.91 \\
\hline Median test 4 & & 8.06 \\
\hline Adjusted BLR & & 8.49 \\
\hline $\mathrm{CPH}$ & & 9.06 \\
\hline Adjusted CPH & & 9.34 \\
\hline Chi-square & & 10.37 \\
\hline Chi-square 4 & & 11.14 \\
\hline Chi-square, fatal stroke & & 12.66 \\
\hline
\end{tabular}

*MWU: Mann-Whitney U test, OLR: Ordinal logistic regression: CA Trend: Cochran-Armitage Trend test, MR: Multiple linear regression, BLR: Binary logistic regression, $\mathrm{CPH}$ : Cox proportional hazards model.

\section{RESULTS}

- Data from 35 trials (257,749 patients) were shared with the collaboration: 13 trials had multiple comparator arms, resulting in 59 datasets.

- The most commonly shared outcome was stroke.

- Using the severity data provided, a 4-level stroke outcome was derived (Fatal/Non-fatal/TIA/None) for 35 datasets.

\section{CONCLUSIONS}

- Methods that are used when analysing polychotomised outcomes appear to be more efficient than those used for binary outcomes.

- This finding is to be further tested for other vascular event outcomes (myocardial infarction, bleeding and composite) using data from the OA-Prevention collaboration. 